\title{
HAEMOcare: The First International Epidemiological Study Measuring Burden of Hemophilia in Developing Countries
}

Naresh Gupta ${ }^{1}$ Abderrahmane Benbouzid ${ }^{2}$ Meriem Belhani ${ }^{3} \quad$ Mohammed El Andaloussi ${ }^{4}$ Khadija Maani $^{5}$ Yasser Wali6,7 Soraya Benchikh El Fegoun ${ }^{8}$ Hossam Ali Saad $^{9}$ Johnny Mahlangu ${ }^{10}$

\author{
${ }^{1}$ Department of Medicine and The Haemophilia Centre, Maulana \\ Azad Medical College \& Associated Lok Nayak and GB Pant \\ Hospitals, New Delhi, India \\ 2 Department of Orthopaedics, University Medical Centre of Ben \\ Aknoun, Ben Aknoun, Algeria \\ ${ }^{3}$ Department of Haematology, University Hospital of Beni Messous, \\ Beni Messous, Algeria \\ 4 Paediatric Orthopaedic and Traumatology Service, Abderrahim \\ Harouchi Hospital, Part of the Ibn Rochd University Hospital, \\ Casablanca, Morocco \\ ${ }^{5}$ Department of Paediatrics, Haematology and Oncology Unit, \\ Abderrahim Harouchi University Children's Hospital-Medical \\ School of Casablanca, Casablanca, Morocco \\ ${ }^{6}$ Department of Child Health, Sultan Qaboos University, Muscat, Oman \\ ${ }^{7}$ Department of Paediatrics, Alexandria University, Alexandria, Egypt \\ ${ }^{8}$ Novo Nordisk Health Care AG, Zurich, Switzerland \\ ${ }^{9}$ Novo Nordisk Inc., Plainsboro, New Jersey, United States \\ 10 Haemophilia Comprehensive Care Centre, Charlotte Maxeke \\ Johannesburg Hospital, Department of Molecular Medicine and \\ Haematology, National Health Laboratory Service and Faculty of \\ the Health Sciences, University of Witwatersrand, Johannesburg, \\ South Africa
}

TH Open 2019;3:e190-e199.

\begin{abstract}
Address for correspondence Naresh Gupta, MD, Department of Medicine and The Haemophilia Centre, Maulana Azad Medical College \& Associated Lok Nayak and GB Pant Hospitals, 2-Bahadur Shah Zafar Marg, New Delhi 110002, India (e-mail: doctornaresh@gmail.com).
\end{abstract}

\author{
Abstract \\ Keywords \\ - HAEMOcare \\ - epidemiological \\ study \\ - hemophilia \\ - inhibitors \\ - orthopedic status \\ - quality of life \\ - developing countries
}

Introduction Optimizing hemophilia care remains challenging in developing countries. Burden-of-disease studies are important to develop strategies for improving hemophilia care.

Aim The HAEMOcare study evaluated the factors contributing to hemophilia-related orthopedic disease burden in developing countries.

Methods HAEMOcare was a noninterventional, cross-sectional, epidemiological study conducted in Algeria, India, Morocco, Oman, and South Africa. Male patients with severe hemophilia ( $N=282$ ) aged $\geq 6$ years, without or with inhibitors, being treated on-demand for bleeding were included. Hemophilia-related orthopedic clinical and functional status was assessed using the Hemophilia Joint Health Score (HJHS), radiological status with the Pettersson Score, and quality of life with the EuroQol five-dimension questionnaire (EQ-5D-3L). Direct and indirect economic costs of hemophilia care were also calculated.

Results Patients (mean [standard deviation, SD] age: 20.8 [10.6] years) experienced a mean annualized bleeding rate of 25.8. Overall mean (SD) HJHS and Pettersson score were 17.9 (12.8) and 15.0 (13.5), respectively; scores were similar between patients received January 24, 2019 accepted

March 15, 2019
DOI https://doi.org/ $10.1055 / \mathrm{s}-0039-1688414$. ISSN 2512-9465. (c) 2019 Georg Thieme Verlag KG Stuttgart · New York
License terms

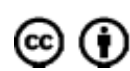


without or with inhibitors ( $p=0.21$ and 0.76 , respectively). Approximately $70 \%$ of adults reported problems relating to pain/discomfort and mobility parameters in the EQ-5D-3L. Mean distance to a hemophilia treatment center (HTC) was $79.4 \mathrm{~km}$. As expected, total costs of hemophilia were statistically significantly higher in patients with inhibitors versus without inhibitors $(p=0.002)$.

Conclusion Inadequate access to HTCs and expert care, along with high bleeding rates, led to equivalent hemophilia-related orthopedic morbidity between hemophilia patients without and with inhibitors. HAEMOcare documented the economic and disease burdens associated with suboptimal hemophilia care in developing countries.

\section{Introduction}

Modern management has significantly improved the clinical course of hemophilia. However, the development of inhibitors against clotting factors and chronic arthropathy remains major causes of morbidity. ${ }^{1}$ In developing countries, with limited resources and limited or no access to any treatment, optimizing hemophilia care services can be beneficial for all patients.

In developed countries, large-scale observational studies have evaluated hemophilia-related orthopedic status and outcomes such as quality of life (QoL) and resource consumption, to consider risk factors for poor outcomes and improve disease management. ${ }^{2-7}$ Few studies have been reported from developing countries. ${ }^{8-10}$ Burden-of-disease studies are needed in developing nations to prioritize determinants of hemophilia care and to formulate adapted management strategies to improve outcomes.

The HAEMOcare study was conducted in five developing countries (defined by World Bank Atlas Method as lowmiddle income ${ }^{11}$ ) to identify the unmet needs of severe hemophilia patients, including exploring the possible relation of hemophilia-related orthopedic status to inhibitors, treatment history, hemophilia management, assessment of QoL, and evaluation of the economic aspects of hemophilia. ${ }^{12}$

\section{Methods}

\section{Study Design}

HAEMOcare (NCT01503567) was a multicenter, noninterventional, cross-sectional, epidemiological study in Algeria, India, Morocco, Oman, and South Africa, with a design similar to a European study. ${ }^{2}$ HAEMOcare was conducted according to the Declaration of Helsinki, Good Clinical Practice as set out by the International Conference on Harmonisation of Technical Requirements for Registration of Pharmaceuticals for Human Use, and applicable national guidelines, with local institutional review board/independent ethics committee approval obtained accordingly. Before enrolment, written informed consent was obtained from each patient, or a legally acceptable representative. Recruitment and studyrelated assessments were conducted over an 8-month period. Every study participant had one visit which included all study-related assessments.

\section{Study Population}

Eligible patients were enrolled between January 2, 2012 and September 3, 2012. Included patients were males aged $\geq 6$ years, with severe congenital hemophilia A or B (FVIII or FIX levels $<1$ international unit [IU]/dL or $<1 \%$ of normal), without or with inhibitors and receiving hemostatic treatment on-demand. Those with other known clinically relevant coagulation disorders, receiving prophylactic hemophilia treatment, or receiving treatment for hepatitis $\mathrm{C}$ or human immunodeficiency virus infection were excluded.

The enrolment target was 300 patients, with 50 to 70 from each participating country. Subgroup analyses were stratified by age and inhibitor status: pediatric patients (6-18 years) without inhibitors; pediatric patients with inhibitors; adult patients ( $>18$ years) without inhibitors; and adult patients with inhibitors.

\section{Objectives}

The primary objective of HAEMOcare was to evaluate the orthopedic status and degree of arthropathy in severe hemophilia A and B patients without or with inhibitors in developing countries. Hemophilia-related orthopedic status was assessed clinically, using the Hemophilia Joint Health Score (HJHS) administered by a physical therapist, and radiologically, using the Pettersson Score; higher scores represented a worse status in both scales.

Secondary objectives were to evaluate the relationship of previous disease management to current disease status, patient QoL, and the economic burden associated with hemophilia treatment. The generic EuroQol five-dimension questionnaire (three-level version; EQ-5D-3L), including the 100 -point visual analogue scale (VAS), was used to assess QoL. ${ }^{13}$ Economic burden was determined for the 12 months before the study visit by measuring direct expenses (treatment and transportation costs), indirect expenses (lost patient/family productivity), and capacity to cover expenses (insurance status; socioeconomic status of the patient/ family) using a predesigned, structured questionnaire. The primary investigator at each site determined occupational and educational status locally, per country standards. Home treatment (full or partial) was at the discretion of the treating physician. Target joints were commonly identified per the International Society on Thrombosis and Hemostasis (ISTH) 
definition as joints in which three or more spontaneous bleeds had occurred within a 6 -month period. ${ }^{14}$

\section{Statistical Analyses}

The prespecified enrolment targets would allow detection of an effect size of 0.40 in the Pettersson score, with a two-sided $\alpha$ of 0.05 and $90 \%$ power, and an effect size of 0.5 with a power $>80 \%$ when controlling for $\leq 8$ covariates in a multiple regression model. Missing data were not imputed; however, when X-rays were unavailable for assessment of Pettersson score, results were imputed as 0 . Results were comparable between observed and imputed Pettersson scores for all analyses, therefore data for observed Pettersson score are reported here.

The association of inhibitor status with Pettersson score, HJHS, and EQ-5D-3L VAS was analyzed using a multivariate linear regression model, adjusting for age, insurance status, access to expert orthopedic consultation, regular sports participation, and regular physical therapy sessions. Direct and indirect economic burdens were assessed with a general linear model adjusting for age, inhibitor status, Pettersson score, and HJHS. Descriptive values are reported as mean with standard deviation (SD). Values from statistical analysis are reported as adjusted estimated difference (AED) and 95\% confidence interval $(\mathrm{CI})$ for Pettersson score and HJHS, while EQ-5D-3L VAS scores are reported as adjusted mean difference (AMD) and 95\% CI. SAS 9.1 software (SAS Institute, Cary, NC, United States) was used for all analyses.

\section{Results}

\section{Study Population}

Patient demographics are shown in - Table 1. Of 282 patients enrolled (250 [88.6\%] hemophilia A, 32 [11.4\%] hemophilia B), 50 (17.7\%) patients had inhibitors. The pediatric group (618 years) included 128 patients, $104(81.2 \%)$ patients without and 24 (18.8\%) with inhibitors; the adult group included 154 patients, 128 (83.1\%) patients without and 26 (16.9\%) with inhibitors. Family history of inhibitors was more common in patients with inhibitors than those without. Hemophilia was diagnosed at a mean age of 34 months, and the presence of inhibitors at a mean age of 175 months (14.6 years).

\section{Orthopedic Assessments}

Mean (SD) total HJHS, HJHS global gait scores, and observed Pettersson scores are shown in - Table 2. Hemophilia-related orthopedic disabilities limited the HJHS in $70 \%$ of patients. Mean overall AED in HJHS was similar between patients without and with inhibitors (AED: -2.45 [95\% CI: -6.30 , 1.40]; $p=0.21 ;$ - Fig. 1), with no significant differences in bilateral HJHS at the ankle, elbow, or knee (-Fig. 2). Overall mean (SD) HJHS global gait score was also similar between patients without and with inhibitors (1.54 [1.32] and 1.35 [1.38], respectively). In addition, overall mean observed Pettersson score was similar between patients without and with inhibitors (AED: 0.72 [95\% CI: -3.91, 5.35]; $p=0.76$ ), and across subgroups ( $\mathbf{- F i g .} \mathbf{3}$ ). There were no significant differences in observed Pettersson scores between any of the patient subgroups (-Fig. 4).

Mean (SD) HJHS was significantly lower in pediatric patients (10.19 [1.57]) than in adults (17.25 [1.58]; AED: 7.05 [95\% CI: 4.08, 10.00]; $p<0.001$ ). Mean (SD) observed Pettersson score was also significantly lower in pediatric patients (9.41 [1.80]) than in adults (15.41 [1.81]; AED: 6.00 [95\% CI: 2.47, 9.53]; $p<0.001$ ).

Of 281 patients assessed, 236 (84\%) patients had target joints; the most common target joints were the knees (right, $53.4 \%$; left, $52.2 \%$ ). Mean annualized bleeding rate (ABR) was 25.8 and similar between patients without or with inhibitors $(p>0.05)$.

\section{Management of Hemophilia}

In the 12 months prior to assessment, 206/232 patients without inhibitors (88\%) received on-demand treatment with FVIII or FIX. Of 50 patients with inhibitors, 21 patients (42\%) reported use of activated recombinant factor VII (rFVIIa), 10 patients (19\%, all adults) reported use of activated prothrombin complex concentrate (aPCC), and 19 patients (38\%) reported use of FVIII concentrates, including ongoing immune tolerance induction (ITI) in one pediatric patient. Some patients reported the use of multiple agents and one pediatric patient without inhibitors received a single dose of rFVIIa $90 \mu \mathrm{g} / \mathrm{kg}$ (-Table 3).

Mean (SD) doses of hemostatic treatment during the 12 months prior to enrolment are reported in -Table 3 . Among adult patients with inhibitors, patients were treated mainly with bypassing agents rFVIIa (46\%) and aPCC (38\%). In pediatric patients with inhibitors, rFVIIa was the only bypassing agent used (nine patients [38\%]).

Of 280 patients with a medical history available, 26 (9.3\%) had any history of prophylaxis prior to enrolment. Prophylaxis was received by $4 / 104$ (3.8\%) pediatric patients without inhibitors, $1 / 24(4.2 \%)$ pediatric patients with inhibitors, $1 / 26$ (3.9\%) adults with inhibitors, and 20/126 (15.9\%) adults without inhibitors. History of previous prophylaxis was similar between patients without inhibitors (24/230 [10.4\%]) and those with inhibitors prior to inhibitor development $(2 / 50$ [4.0\%]; $p=0.19$ ). No patients with inhibitors had received any prophylaxis with bypassing agents.

There was no difference in the proportion of hemophiliarelated orthopedic surgical procedures $(p=0.97)$, use of orthopedic aids ( $p=0.44$ ), regular physical therapy sessions $(p=0.86)$, or regular participation in sport $(p=0.28)$ between patients without and with inhibitors. More patients with inhibitors $(35 ; 71.4 \%)$ received prompt hemostatic treatment within the first 2 hours of bleeding compared with those without (123; 53.3\%; $p=0.03 ;-$ Table 4).

Thirty-seven percent of patients received home treatment; a greater proportion $(25 / 50 ; 50 \%)$ of patients with inhibitors practiced home treatment than those without (80/ 232; 35\%; $p=0.04 ;-$ Table 4). Patients travelled a mean(SD) of $79.4(124.0) \mathrm{km}$ to reach their hemophilia treatment center (HTC), incurring a mean (SD) transportation cost of US\$13.02 (47.9) per visit. Regular physician follow-up was feasible for $234 / 282$ (83.0\%) patients. Mean (SD) number of 
Table 1 Demographic characteristics of the HAEMOcare study population

\begin{tabular}{|c|c|c|c|c|c|}
\hline & \multicolumn{2}{|c|}{ Pediatric (6-18 y) } & \multicolumn{2}{|l|}{ Adult (>18 y) } & \multirow[b]{2}{*}{$\begin{array}{l}\text { Total } \\
(N=282)\end{array}$} \\
\hline & $\begin{array}{l}\text { Without } \\
\text { inhibitors } \\
(n=104)\end{array}$ & $\begin{array}{l}\text { With } \\
\text { inhibitors } \\
(n=24)\end{array}$ & $\begin{array}{l}\text { Without } \\
\text { inhibitors } \\
(n=128)\end{array}$ & $\begin{array}{l}\text { With } \\
\text { inhibitors } \\
(n=26)\end{array}$ & \\
\hline \multicolumn{6}{|l|}{ Country of enrolment, $n$ (\%) } \\
\hline Algeria & $11(11)$ & $7(29)$ & $32(25)$ & $10(38)$ & $60(21)$ \\
\hline India & $31(30)$ & $4(17)$ & $40(31)$ & $5(19)$ & $80(28)$ \\
\hline Morocco & $43(41)$ & $7(29)$ & $10(8)$ & 0 & $60(21)$ \\
\hline Oman & $19(18)$ & $6(25)$ & $26(20)$ & $2(8)$ & $53(19)$ \\
\hline South Africa & 0 & 0 & $20(16)$ & $9(35)$ & $29(10)$ \\
\hline Mean age at enrolment, years (SD) & $11.8(3.6)$ & $12.3(3.8)$ & $28.5(9.2)$ & $27.0(5.8)$ & $20.8(10.6)$ \\
\hline \multicolumn{6}{|l|}{ Race, $n(\%)$} \\
\hline White & $54(52)$ & $14(58)$ & $48(38)$ & $12(46)$ & $128(45)$ \\
\hline Black/African & 0 & 0 & $14(11)$ & $6(23)$ & $20(7)$ \\
\hline Asian & $31(30)$ & $4(17)$ & $41(32)$ & $6(23)$ & $82(29)$ \\
\hline Other & $19(18)$ & $6(25)$ & $25(20)$ & $2(8)$ & $52(18)$ \\
\hline \multicolumn{6}{|l|}{ Marital status, $n(\%)$} \\
\hline Married & 0 & 0 & $27(29)$ & $3(12)$ & $40(14)$ \\
\hline Unmarried & $104(100)$ & $24(100)$ & $91(71)$ & $23(88)$ & $242(86)$ \\
\hline \multicolumn{6}{|c|}{ Occupational and educational status, ${ }^{\mathrm{a}} \mathrm{n}(\%)$} \\
\hline Above local average & $33(32)$ & $8(33)$ & $33(26)$ & $7(27)$ & $81(29)$ \\
\hline Local average & $51(49)$ & $13(54)$ & $49(38)$ & $7(27)$ & $120(43)$ \\
\hline Below local average & $20(19)$ & $3(13)$ & $46(36)$ & $12(46)$ & $81(29)$ \\
\hline \multicolumn{6}{|l|}{ Family history of hemophilia, $n$ (\%) } \\
\hline Yes & $66(63)$ & $17(71)$ & $91(71)$ & $19(73)$ & $193(68)$ \\
\hline No & $38(37)$ & $7(29)$ & 37 (29) & $7(27)$ & $89(32)$ \\
\hline Family history of inhibitors, $n$ (\%) & $(n=66)$ & $(n=17)$ & $(n=91)$ & $(n=19)$ & $(n=193)$ \\
\hline Yes & $6(9)$ & $5(29)$ & $3(3)$ & $6(32)$ & $20(10)$ \\
\hline No & $58(88)$ & $10(59)$ & $86(95)$ & $12(63)$ & $166(86)$ \\
\hline Unknown & $2(3)$ & $2(12)$ & $2(2)$ & $1(5)$ & $7(4)$ \\
\hline \multicolumn{6}{|l|}{ Type of hemophilia, n (\%) } \\
\hline A & $96(92)$ & $24(100)$ & $104(81)$ & $26(100)$ & $250(89)$ \\
\hline $\mathrm{B}$ & $8(8)$ & 0 & $24(19)$ & 0 & $32(11)$ \\
\hline $\begin{array}{l}\text { Mean time since diagnosis, } \\
\text { months }(\mathrm{SD})\end{array}$ & $\begin{array}{l}(n=100) \\
121.3(43.1)\end{array}$ & $\begin{array}{l}(n=23) \\
138.2(44.2)\end{array}$ & $\begin{array}{l}(n=123) \\
300.2(122.1)\end{array}$ & $\begin{array}{l}(n=22) \\
287.6(97.8)\end{array}$ & $\begin{array}{l}(n=268) \\
218.5(126.4)\end{array}$ \\
\hline $\begin{array}{l}\text { Mean time since diagnosis } \\
\text { of inhibitors, months (SD) }\end{array}$ & - & $\begin{array}{l}(n=24) \\
36.6(27.5)\end{array}$ & - & $\begin{array}{l}(n=25) \\
85.8(68.4)\end{array}$ & $\begin{array}{l}(n=49) \\
61.7(57.6)\end{array}$ \\
\hline $\begin{array}{l}\text { Average bleeds per month } \\
\text { during prior } 12 \text { months, mean (SD) }\end{array}$ & $\begin{array}{l}(n=86) \\
2.0(1.8)\end{array}$ & $\begin{array}{l}(n=22) \\
2.0(1.7)\end{array}$ & $\begin{array}{l}(n=124) \\
2.3(2.0)\end{array}$ & $\begin{array}{l}(n=26) \\
2.0(1.5)\end{array}$ & $\begin{array}{l}(n=258) \\
2.2(1.9)\end{array}$ \\
\hline Presence of target joints, $n(\%)$ & $89(86)$ & $18(75)$ & $112(88)$ & $17(65)$ & $236(84)$ \\
\hline \multicolumn{6}{|l|}{ Insurance/incapacity benefits, $n$ (\%) } \\
\hline Fully reimbursed & $37(36)$ & $15(63)$ & $71(55)$ & $16(62)$ & 139 (49) \\
\hline Partially reimbursed & $10(10)$ & $3(13)$ & $7(5)$ & $2(8)$ & $22(8)$ \\
\hline Not reimbursed & $57(55)$ & $6(25)$ & $50(39)$ & $8(31)$ & $121(43)$ \\
\hline
\end{tabular}

${ }^{a}$ The primary investigator, according to country standards, determined average occupational and educational status locally. 
e194 HAEMOcare: Hemophilia in Developing Countries Gupta et al.

Table 2 Summary of mean total Pettersson score, HJHS, and global gait assessment (HJHS)

\begin{tabular}{|c|c|c|c|c|c|}
\hline & \multicolumn{2}{|l|}{ Pediatric (6-18 y) } & \multicolumn{2}{|l|}{ Adult $(>18 \mathrm{y})$} & \multirow[b]{2}{*}{$\begin{array}{l}\text { Total } \\
(N=282)\end{array}$} \\
\hline & $\begin{array}{l}\text { Without inhibitors } \\
(n=104)\end{array}$ & $\begin{array}{l}\text { With inhibitors } \\
(n=24)\end{array}$ & $\begin{array}{l}\text { Without inhibitors } \\
(n=128)\end{array}$ & $\begin{array}{l}\text { With inhibitors } \\
(n=26)\end{array}$ & \\
\hline $\begin{array}{l}\text { Observed Pettersson } \\
\text { score, mean (SD) }\end{array}$ & $\begin{array}{l}(n=85) \\
12.3(13.3)\end{array}$ & $\begin{array}{l}(n=20) \\
14.2(19.6)\end{array}$ & $\begin{array}{l}(n=104) \\
17.0(12.6)\end{array}$ & $\begin{array}{l}(n=19) \\
16.8(9.7)\end{array}$ & $\begin{array}{l}(n=228) \\
15.0(13.5)\end{array}$ \\
\hline Total HJHS, mean (SD) & $\begin{array}{l}(n=102) \\
15.2(12.2)\end{array}$ & $\begin{array}{l}(n=23) \\
10.9(13.0)\end{array}$ & $\begin{array}{l}(n=127) \\
20.8(13.1)\end{array}$ & $\begin{array}{l}(n=26) \\
20.2(9.1)\end{array}$ & $\begin{array}{l}(n=278) \\
17.9(12.8)\end{array}$ \\
\hline \multicolumn{6}{|l|}{$\begin{array}{l}\text { Skills not within normal } \\
\text { limits, } n(\%)\end{array}$} \\
\hline 0 & $47(45.2)$ & $15(62.5)$ & $20(15.6)$ & $3(11.5)$ & $85(30.1)$ \\
\hline 1 & $20(19.2)$ & $4(16.7)$ & 28 (21.9) & 7 (26.9) & 59 (20.9) \\
\hline 2 & $22(21.2)$ & $1(4.2)$ & 37 (28.9) & 7 (26.9) & $67(23.8)$ \\
\hline 3 & $7(6.7)$ & $1(4.2)$ & $21(16.4)$ & $5(19.2)$ & $34(12.1)$ \\
\hline 4 & $5(4.8)$ & $1(4.2)$ & $20(15.6)$ & $4(15.4)$ & $30(10.6)$ \\
\hline Not applicable/missing & $3(2.9)$ & $2(8.3)$ & $2(1.6)$ & 0 & $7(2.5)$ \\
\hline
\end{tabular}

Abbreviations: HJHS, Hemophilia Joint Health Score; SD, standard deviation.

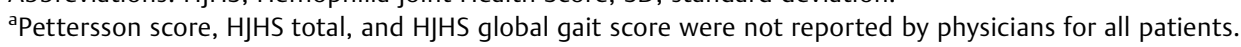

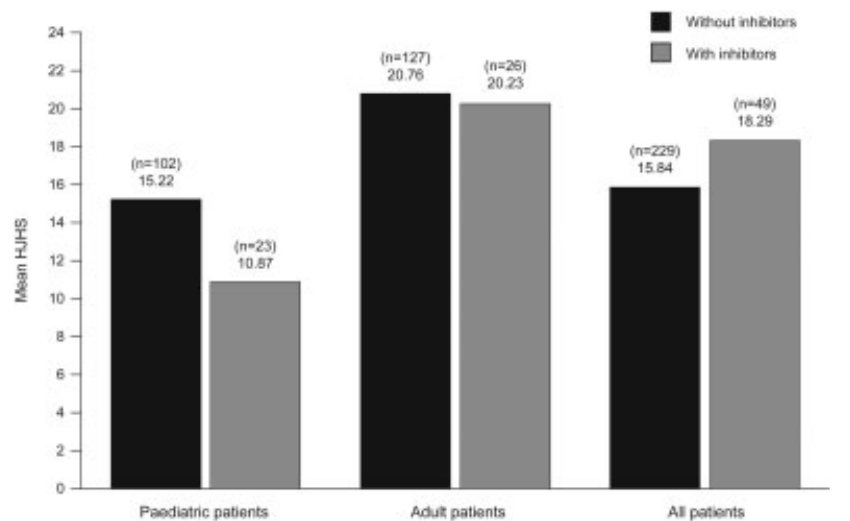

Fig. 1 Mean total Hemophilia Joint Health Score in patients without and with inhibitors.

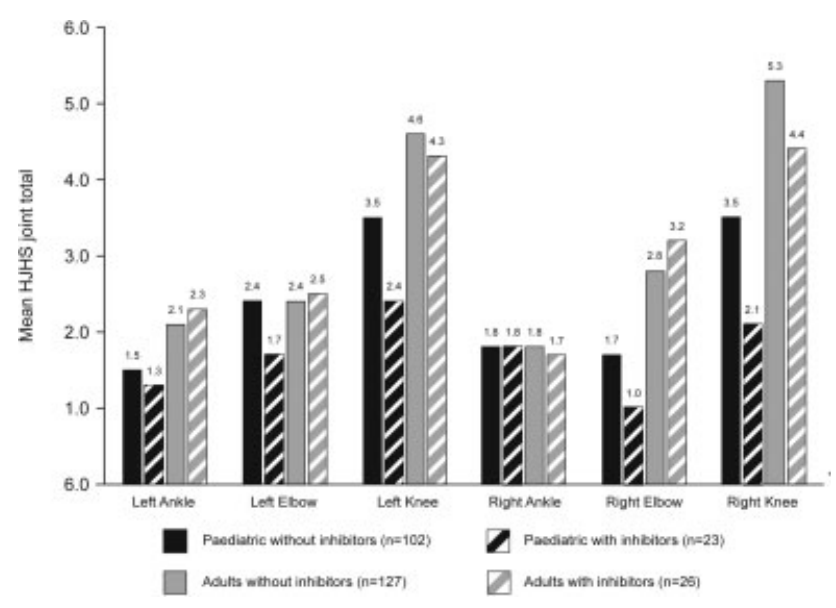

Fig. 2 Mean HJHS joint scores in patients without and with inhibitors. HJHS, Hemophilia Joint Health Score.

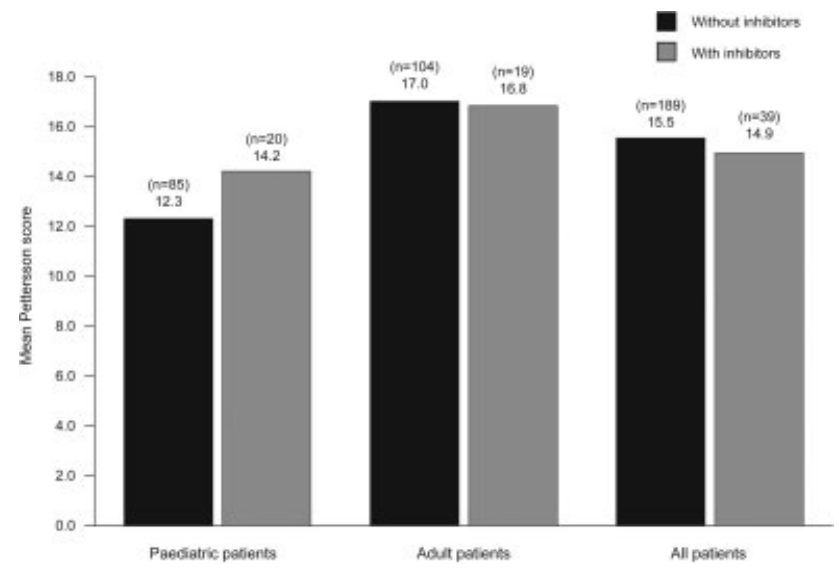

Fig. 3 Mean observed Pettersson score among patients without and with inhibitors.

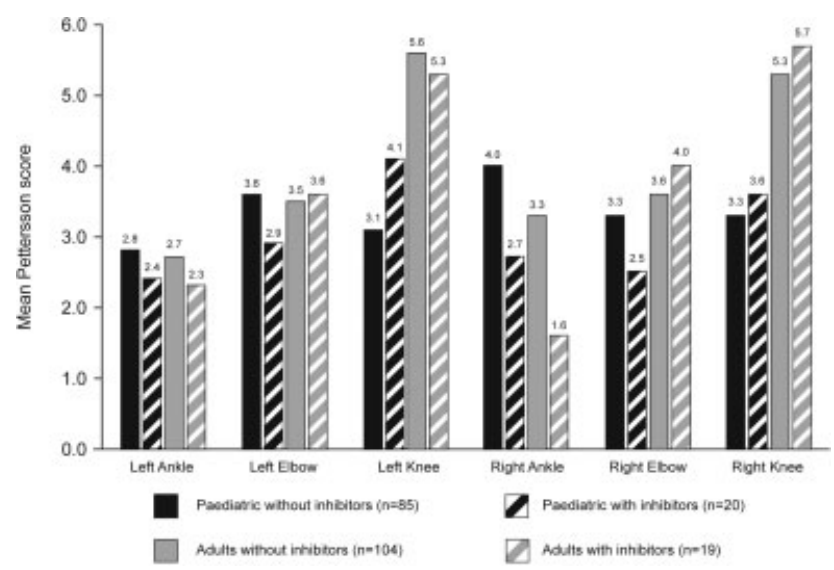

Fig. 4 Mean observed Pettersson joint scores in patients without and with inhibitors. 
Table 3 Summary of antihemophilia therapy doses administered to patients in the 12 months prior to enrolment ${ }^{\mathrm{a}}$

\begin{tabular}{|c|c|c|c|}
\hline Patients without inhibitors & Pediatric $(n=104)$ & Adult $(n=128)$ & Total $(n=232)$ \\
\hline \multicolumn{4}{|l|}{ FVIII (IU/kg) } \\
\hline$n(\%)$ & $81(78)$ & $100(78)$ & $181(79)$ \\
\hline Mean (SD) & $4,172.26(9,462.27)$ & $20,698.59(67,070.87)$ & $13,302.83(50,812.12)$ \\
\hline \multicolumn{4}{|l|}{$\mathrm{FIX}(\mathrm{IU} / \mathrm{kg})$} \\
\hline$n(\%)$ & $4(4)$ & $21(16)$ & $25(11)$ \\
\hline Mean (SD) & $3,469.50(3,540.1)$ & $15,260.19(28,541.23)$ & $13,373.68(26,454.94)$ \\
\hline Patients with inhibitors & Pediatric $(n=24)$ & Adult $(n=26)$ & Total $(n=50)$ \\
\hline \multicolumn{4}{|l|}{ rFVIIa $(\mu \mathrm{g} / \mathrm{kg})$} \\
\hline$n(\%)$ & $9(38)$ & $12(46)$ & $21(42)$ \\
\hline Mean (SD) & $2,316.22(2,548.46)$ & $16,141.00(34,074.10)$ & $10,216.10(26,273.91)$ \\
\hline \multicolumn{4}{|l|}{$\mathrm{aPCC}(\mathrm{IU} / \mathrm{kg})$} \\
\hline$n(\%)$ & - & $10(38)$ & $10(19)$ \\
\hline Mean (SD) & - & $28,536(34,618.22)$ & $28,536.00(34,618.22)$ \\
\hline \multicolumn{4}{|l|}{ FVIII (IU/kg) } \\
\hline$n(\%)$ & $13(54)$ & $6(23)$ & $19(38)$ \\
\hline Mean (SD) & $1,614.77(1,449.57)$ & $1,156.17(1520)$ & $1,469.95(1,445.89)$ \\
\hline
\end{tabular}

Abbreviations: aPCC, activated prothrombin complex concentrate; IU, international unit; rFVIla, activated recombinant factor VII; SD, standard deviation.

Note: one pediatric patient without inhibitors received a single dose rFVlla $90 \mu \mathrm{g} / \mathrm{kg}$ and nine patients without and one patient with inhibitors received "other" hemophilia treatment (specific details of treatment not recorded).

${ }^{a}$ Numbers reported in the table do not match the total values for each group due to missing data and some patients receiving multiple therapies.

Table 4 Management of hemophilia in patients without and with inhibitors

\begin{tabular}{|c|c|c|c|c|}
\hline & $\begin{array}{l}\text { Without inhibitors } \\
(n=232)\end{array}$ & $\begin{array}{l}\text { With inhibitors } \\
(n=50)\end{array}$ & $\begin{array}{l}\text { Total } \\
(N=282)\end{array}$ & $p$-Value \\
\hline Practice home treatment, $n$ (\%) & $80(34.5)$ & $25(50)$ & $105(37.2)$ & 0.04 \\
\hline Hemostatic treatment initiated $<2 \mathrm{~h}$ after bleed onset, $n(\%)$ & $123(53.3)$ & $35(71.4)$ & $158(56.0)$ & 0.03 \\
\hline Orthopedic surgical procedures (any type), $n$ (\%) & $33(14.2)$ & $7(14.0)$ & $40(14.2)$ & 0.97 \\
\hline Use of orthopedic aid (any type), $n(\%)^{a}$ & $102(44.0)$ & $25(50.0)$ & $127(45.0)$ & 0.44 \\
\hline Regular physical therapy sessions, $n(\%)$ & $99(42.7)$ & $22(44.0)$ & $121(42.9)$ & 0.86 \\
\hline Regular participation in sport, $n(\%)$ & $51(22.0)$ & $6(12.0)$ & $57(20.2)$ & 0.28 \\
\hline
\end{tabular}

aDuring the 12 months prior to assessment.

visits to the nearest HTC during the preceding 12 months was 12.8 (14.8), against an ABR of 25.8. An orthopedic expert was available to $133 / 282(47.2 \%)$ patients on a regular basis and to $90(31.9 \%)$ patients irregularly (-Table 5).

\section{Relationships between Hemophilia-Related Orthopedic Status and Hemophilia Management}

Mean observed Pettersson score and HJHS were similar for patients with or without: access to home care, access to expert orthopedic care, regular participation in sports, receipt of regular physical therapy (-Table 5).

\section{Quality of Life}

Most patients reported problems on the EQ-5D-3L domains, most often in the pain/discomfort (any problems, 65.2\%) and mobility (any problems, 56.4\%) dimensions. Reported problems were similar between pediatric and adult patients (-Fig. 5). Mean EQ-5D-3L VAS scores were similar between patients without inhibitors (68.74) and with inhibitors (73.54; AMD: 3.78 [95\% CI: -3.00, 10.56]; $p=0.27$ ). Mean (SD) VAS scores were 67.37 (19.45) in adults without inhibitors, 70.65 (19.70) in adults with inhibitors, 70.43 (24.62) in pediatric patients without inhibitors, and 76.67 (24.12) in pediatric patients with inhibitors.

\section{Economic Aspects of Hemophilia}

Socioeconomic data are shown in -Table 1. Occupational and educational status was average or higher for $71 \%$ of patients. Overall, mean (SD) family income/month was US $\$ 907.60$ (1,246.01; median, US\$350 [range, 15-7,772]) and 
Table 5 Summary of HJHS and Pettersson scores by hemophilia care situation $^{\mathrm{a}}$

\begin{tabular}{|l|l|l|}
\hline & $\begin{array}{l}\text { Pettersson } \\
\text { score } \\
\text { (observed) }\end{array}$ & HJHS \\
\hline $\begin{array}{l}\text { Home treatment, } \\
\text { mean (SD) }\end{array}$ & $(n=228)$ & $(n=278)$ \\
\hline Yes $(n=105)$ & $18.49(14.86)$ & $19.75(13.62)$ \\
\hline No $(n=177)$ & $13.37(12.50)$ & $16.74(12.21)$ \\
\hline $\begin{array}{l}\text { Regular physical } \\
\text { therapy, mean (SD) }\end{array}$ & $(n=228)$ & $(n=278)$ \\
\hline Yes $(n=121)$ & $14.29(13.41)$ & $17.90(12.01)$ \\
\hline No $(n=161)$ & $15.64(13.56)$ & $17.82(13.43)$ \\
\hline $\begin{array}{l}\text { Regular sport, } \\
\text { mean }(\text { SD) }\end{array}$ & $(n=228)$ & $(n=278)$ \\
\hline Yes $(n=57)$ & $10.83(10.49)$ & $14.88(11.07)$ \\
\hline No $(n=224)$ & $16.06(13.97)$ & $18.69(13.13)$ \\
\hline Nonparticipant $(n=1)$ & - & 5.00 \\
\hline $\begin{array}{l}\text { Access to an orthopedic } \\
\text { expert, mean (SD) }\end{array}$ & $(n=227)$ & $(n=275)$ \\
\hline Regular $(n=133)$ & $17.79(15.66)$ & $19.73(13.74)$ \\
\hline \begin{tabular}{l} 
Irregular $(n=90)$ \\
\hline No $(n=56)$
\end{tabular} & $12.91(9.90)$ & $17.81(11.41)$ \\
\hline
\end{tabular}

Abbreviations: HJHS, Hemophilia Joint Health Score; SD, standard deviation. aPettersson score, HJHS and access to an orthopaedic expert were not reported by physicians for all patients.

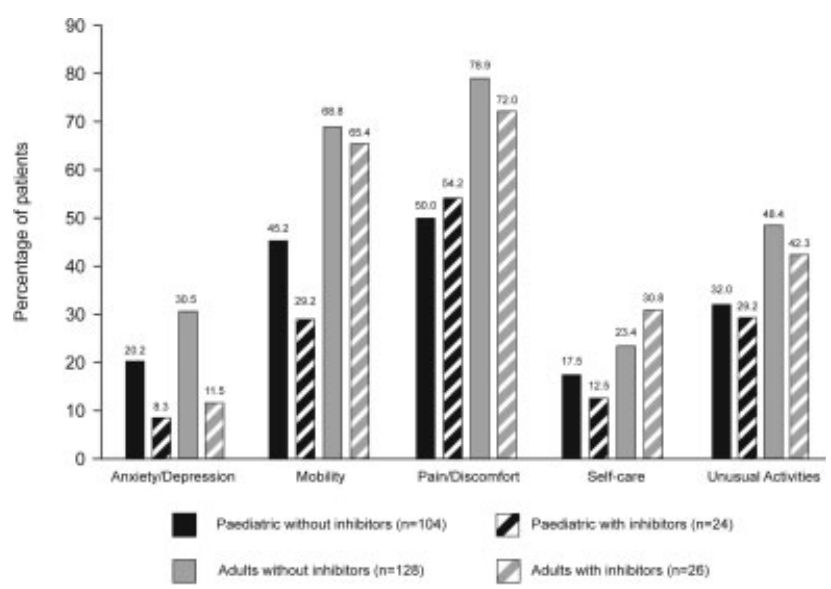

Fig. 5 Percentage of hemophilia patients who reported having problems according to the dimensions of the EQ-5D-3L questionnaire.

treatment costs were fully reimbursed in $139 / 282$ patients (49.3\%), and partially reimbursed in $22 / 282$ patients (7.8\%). In Algeria, all patients, regardless of age or inhibitor status, had treatment costs fully reimbursed. In Oman, all pediatric patients, without or with inhibitors, and adult patients with inhibitors had treatment costs fully reimbursed. No reimbursement was available for 121 patients (42.9\%); the majority of these 121 patients were from India (64 patients) and Morocco (41 patients).

Mean monthly direct ( - Table 6 ) and indirect ( - Table 7 ) costs relating to hemophilia treatment during the 12 months
Table 6 Direct monthly costs (in US\$) associated with hemostatic treatment in patients without and with inhibitors $(n=255)^{\mathrm{a}}$

\begin{tabular}{|c|c|c|c|}
\hline & $\begin{array}{l}\text { Without } \\
\text { inhibitors }\end{array}$ & $\begin{array}{l}\text { With } \\
\text { inhibitors }\end{array}$ & Total \\
\hline \multicolumn{4}{|l|}{ FVIII } \\
\hline$n$ & 177 & 19 & 196 \\
\hline Mean (SD) & $\begin{array}{l}1,566.36 \\
(2,389.25)\end{array}$ & $\begin{array}{l}1,230.79 \\
(1,634.91)\end{array}$ & $\begin{array}{l}1,533.83 \\
(2,325.71)\end{array}$ \\
\hline \multicolumn{4}{|l|}{ FIX } \\
\hline$n$ & 24 & - & 24 \\
\hline Mean (SD) & $\begin{array}{l}952.67 \\
(1151.32)\end{array}$ & - & $\begin{array}{l}952.67 \\
(1151.32)\end{array}$ \\
\hline \multicolumn{4}{|l|}{ rFVIIa } \\
\hline$n$ & 1 & 16 & 17 \\
\hline Mean (SD) & 71.0 & $\begin{array}{l}6,424.88 \\
(8,977.63)\end{array}$ & $\begin{array}{l}6,051.12 \\
(8,828.09)\end{array}$ \\
\hline \multicolumn{4}{|l|}{ aPCC } \\
\hline$n$ & - & 7 & 7 \\
\hline Mean (SD) & - & $\begin{array}{l}2,141.00 \\
(2,203.45)\end{array}$ & $\begin{array}{l}2,141.00 \\
(2,203.45)\end{array}$ \\
\hline \multicolumn{4}{|l|}{ Other } \\
\hline$n$ & 9 & 2 & 11 \\
\hline Mean (SD) & $\begin{array}{l}25.44 \\
(29.05)\end{array}$ & $\begin{array}{l}20.00 \\
(15.56)\end{array}$ & $\begin{array}{l}24.45 \\
(26.53)\end{array}$ \\
\hline
\end{tabular}

Abbreviations: aPCC, activated prothrombin complex concentrate; rFVIlla, activated recombinant factor VII; SD, standard deviation.

${ }^{a}$ Direct monthly costs were not reported by physicians for all patients.

Table 7 Indirect consumption of patient/family and community resources during the 12 months before enrolment

\begin{tabular}{|c|c|c|}
\hline & Without inhibitors & With inhibitors \\
\hline \multicolumn{3}{|c|}{ Days of school absenteeism (patient) } \\
\hline$N$ & 153 & 33 \\
\hline Mean (SD) & $29.7(36.7)$ & $32.0(36.1)$ \\
\hline \multicolumn{3}{|c|}{ Working days lost (patient) } \\
\hline$n$ & 42 & 8 \\
\hline Mean (SD) & $30.8(38.2)$ & $69.5(61.9)$ \\
\hline \multicolumn{3}{|c|}{ Indirect cost of lost working days (US\$, patient) } \\
\hline$n$ & 38 & 7 \\
\hline Mean (SD) & $440.9(781.9)$ & $3,192.6(4,220.9)$ \\
\hline \multicolumn{3}{|c|}{ Working days lost (family) } \\
\hline$n$ & 59 & 12 \\
\hline Mean (SD) & $19.7(15.2)$ & $25.8(40.1)$ \\
\hline \multicolumn{3}{|c|}{ Indirect cost of lost working days (US\$) } \\
\hline$n$ & 58 & 11 \\
\hline Mean (SD) & $344.0(810.9)$ & $415.6(541.3)$ \\
\hline
\end{tabular}

Abbreviation: SD, standard deviation.

preceding enrolment were used to compute the combined annualized cost of managing hemophilia. The total combined mean (SD) annualized cost of hemophilia was US $\$ 19,513$ 
$(38,739)$ per year, ranging from $\$ 9,135(12,196)$ for pediatric patients without inhibitors to $\$ 47,225(91,203)$ for adults with inhibitors. Costs were significantly lower for patients without inhibitors than those with inhibitors for direct (AED: US\$18,154.50 [95\% CI: 6,371.71-29,937.29]; $p=0.003$ ), indirect (AED: US\$999.68 [95\% CI: 277.76-1,721.59]; $p=0.007$ ), and total costs (AED: US\$18,551.43 [95\% Cl: $6,791.76-30,311.10 ; p=0.002)$.

\section{Discussion}

The current study assessed hemophilia-related orthopedic status, QoL, and economic burden of patients currently treated on-demand in five countries. HJHS and Pettersson scores were similar between hemophilia patients without and with inhibitors, and both scores were significantly lower in pediatric patients compared with adults. In developed countries, studies have reported better hemophilia-related orthopedic status in patients without inhibitors, including the European study that inspired HAEMOcare. ${ }^{2,15}$ Numerous studies have shown the effect of prophylaxis in improving ABR, joint symptoms, and QoL in patients without inhibitors. $^{15-18}$ The dismal results in HAEMOcare may reflect suboptimal hemophilia care for those without inhibitors in developing countries.

Notably, our study did not collect information on treatment protocols, nor was adherence to a specific/standard protocol required; patients were treated using local protocols, and patients on prophylaxis at the time of study recruitment were not included. Only $11 \%$ of adults and $4 \%$ of pediatric hemophilia patients being treated on-demand had any history of prior prophylactic therapy, considerably lower than rates of prophylaxis reported in developed countries (adults, 38-55\%; pediatric patients, 41-84\%). ${ }^{19,20}$ For patients with inhibitors in our study, only two patients being treated on-demand had ever received prophylaxis and only one patient (pediatric) had received ITI.

In our study, $70 \%$ of patients being treated on-demand reported limitations on the HJHS from hemophilia-related orthopedic morbidities, consistent with previous reports. ${ }^{21}$ Despite the overall young age (mean: 20.8 years) of patients, $84 \%$ had target joints. Patients experienced a mean ABR of 25.8 , with no significant differences between adults and pediatric patients, or between those without and with inhibitors. This finding contrasts with developed countries, where substantially lower bleeding rates have been observed; in a German study, only $17 \%$ of patients receiving on-demand therapy reported more than 12 bleeds per year and $39 \%$ of patients had fewer than one bleed per year. ${ }^{22}$ However, patients without inhibitors receiving on-demand treatment in developed countries could be expected to have only a mild bleeding phenotype, as severely bleeding patients will generally receive prophylaxis. ${ }^{23}$ Therefore, any comparison of the patients in the current study with populations receiving on-demand treatment in developed countries requires caution. Despite this caveat, our results suggest suboptimal hemostatic treatment and orthopedic care in developing countries for patients with hemophilia.
Barriers to early hemostatic treatment of bleeding events can impact hemophilia-related orthopedic disease burden; such barriers include substantial treatment costs, parent/caregiver inconvenience, distance to the HTC, and more rarely, problems related to venous access or home infusion. ${ }^{24}$

The results of the study showed that there is a need for wider and earlier comprehensive care, despite patients in HAEMOcare receiving the best standard of care available to them at the time, as expert orthopedic care was offered mainly to patients with more advanced morbidities. Patients with regular access to orthopedic care showed numerically higher HJHS and Pettersson score than patients without, indicating that access to care may be focused on patients with more severe morbidities or that referrals to an orthopedist are late ( $\mathbf{- T a b l e ~} \mathbf{5}$ ).

Similar QoL scores were found between patients with and without inhibitors. The similarity in QoL among patients, regardless of inhibitor status, may suggest that the level of hemophilia care for patients without inhibitors currently (and in most cases lifelong) treated on-demand was inadequate, and therefore their QoL was similar to that of patients with inhibitors. This similarity contrasts with results previously reported in Europe, in which the absence of inhibitors had a positive impact on QoL. ${ }^{2,25}$

Despite similar QoL, the direct, indirect, and total costs associated with hemophilia and its treatment were, as expected, lower in patients without inhibitors than in those with inhibitors and only $57 \%$ of patients had access to full or partial medical insurance. However, FVIII and FIX concentrates and bypassing agents were not always available or accessible, which may have impacted upon the treatments received and their costs. It is important to note that these costs are purely indicative and were incurred in 2012 and there may be variations between the systems of care in each country that affect the availability and pricing of treatments. While access to hemophilia care follow-up was considered to be feasible in $83 \%$ of patients, the mean travel distance to their HTC was $79.4 \mathrm{~km}$ and mean transportation costs were evaluated to be around \$US13 per visit (1.4\% of the mean family monthly income of \$US 907.60). Wider universal health coverage or individual health insurance coverage would greatly help to reduce the economic burden for hemophilia patients. The availability of treatment close to the patient or at home and referral networks to reduce travel distances would also provide economic and medical benefits to patients.

Home treatment was often initiated late in the disease course, which could explain observations of numerically higher HJHS and Pettersson score for patients who received home treatment (-Table 5). Patients would benefit from wider application and earlier initiation of supervised home treatment programs, as recommended in the World Federation of Hemophilia guidelines. ${ }^{26}$

\section{Limitations}

Patients receiving prophylaxis were excluded from HAEMOcare. It is estimated that only 1 to $31 \%$ of all patients in the study countries would have received prophylaxis at the time of the study, with the exception of Algeria. ${ }^{27}$ Although 
prophylaxis estimates for Algeria had reached $90 \%$ for pediatric and $40 \%$ for adult patients in $2015,{ }^{28}$ it was expected that they would have been similar to other developing countries at the time of the study. Therefore, given the low rate of previous exposure to prophylaxis in patients in this study and the exclusion of patients currently receiving prophylaxis, we believe that the study population here was reflective of the rate of prophylaxis in the population of patients with severe hemophilia at the time of the study, in at least four of the five developing countries studied.

This study was only able to enroll 50 patients with inhibitors against a target of 75 , limiting its statistical power. Furthermore, much of the data were collected retrospectively, and radiological scores were based on preexisting X-rays.

\section{Conclusions}

The HAEMOcare study showed that, in the participating developing countries, inadequate access to HTCs and expert care along with high bleeding rates led to equivalent hemophilia-related orthopedic status, arthropathy, and QoL between patients without and with inhibitors. The economic burden from disease was high in terms of direct and indirect costs, and the large travel distances to access hemophilia treatment and expert consultations added to the burden of hemophilia patients. HAEMOcare documented the economic and disease burdens associated with suboptimal hemophilia care in developing countries.

\section{Authors' Contributions}

Naresh Gupta designed and performed the research, analyzed the data, and wrote and edited the manuscript. Abderrahmane Benbouzid performed the research and wrote and edited the manuscript. Meriem Belhani wrote and edited the manuscript. Mohammed El Andaloussi performed the research and wrote and edited the manuscript. Khadija Maani performed the research and wrote and edited the manuscript. Yasser Wali designed and performed the research, analyzed the data, and wrote and edited the manuscript. Soraya Benchikh El Fegoun designed the research, analyzed the data, and wrote and edited the manuscript. Hossam Ali Saad designed the research, analyzed the data, and wrote and edited the manuscript. Johnny Mahlangu designed and performed the research, analyzed the data, and wrote and edited the manuscript.

\section{Conflict of Interest}

Yasser Wali has received research grants from Novo Nordisk and Pfizer. Soraya Benchikh El Fegoun is an employee of Novo Nordisk Health Care AG. Hossam Ali Saad is an employee of Novo Nordisk Inc. Johnny Mahlangu has received research grants from Bayer, Biogen, CLS, Novo Nordisk, and Roche. He is a member of advisory committees of Baxalta, Biogen, CLS, Novo Nordisk, and Roche, and speaker bureau of Amgen, Bayer, Biogen, Biotest, and CLS. All other authors declare no conflict of interest.

\section{Acknowledgments}

The HAEMOcare study was sponsored by Novo Nordisk. Medical writing support was provided by Sam Hurrell (Bioscript, Macclesfield, United Kingdom), and funded by Novo Nordisk A/S. The authors would like to thank Professor Massimo Morfini (no current affiliation) and Souror Senoussaoui (Novo Nordisk Health Care AG) for their review of the manuscript, and Dr. Ismail Elbeshlawi (Department of Child Health, Sultan Qaboos University, Muscat, Oman, and Department of Pediatrics, Alexandria University, Egypt), Dr Mehdi Chafai (Orthopaedics Department, Abderrahim Harouchi Children's Hospital, Medical School of Casablanca, Casablanca, Morocco), Dr Mohamed Elshinawy (Child Health Department SQUH, Oman), Mr. Mohamed Alshemli (Physiotherapy Department, SQUH, Oman), and Dr Bensadok Meriem (Department of Haematology, University Hospital of Benimessous, Algiers) for their contributions to this project.

\section{References}

1 Raffini L, Manno C. Modern management of haemophilic arthropathy. Br J Haematol 2007;136(06):777-787

2 Morfini M, Haya S, Tagariello G, et al. European study on orthopaedic status of haemophilia patients with inhibitors. Haemophilia 2007;13(05):606-612

3 Tusell JM, Aznar JA, Querol F, Quintana M, Moreno M, Gorina E; Orthopaedic Study Group. Results of an orthopaedic survey in young patients with severe haemophilia in Spain. Haemophilia 2002;8(Suppl 2):38-42

4 Soucie JM, Cianfrini C, Janco RL, et al. Joint range-of-motion limitations among young males with hemophilia: prevalence and risk factors. Blood 2004;103(07):2467-2473

5 Windyga J, Stefańska E, Lopaciuk S, Juszyński A, Woźniak D, Strzelecki $O$. The orthopaedic status of a selected severe haemophilia group [in Polish]. Pol Arch Med Wewn 2005;113(06):562-569

6 Morfini M. Articular status of haemophilia patients with inhibitors. Haemophilia 2008;14(Suppl 6):20-22

7 Stieltjes N, Torchet MF, Misrahi L, et al. Epidemiological survey of haemophiliacs with inhibitors in France: orthopaedic status, quality of life and cost-the "Statut Orthopédique des Patients Hémophiles" avec Inhibiteur study. Blood Coagul Fibrinolysis 2009;20(01):4-11

8 Ferreira AA, Leite IC, Bustamante-Teixeira MT, Guerra MR. Hemophilia A in Brazil-epidemiology and treatment developments. J Blood Med 2014;5:175-184

9 Haghpanah S, Mohtadi H, Akbari M, Karimi M. Quality of life in children and adolescents with rare bleeding disorders in southern Iran. Clin Appl Thromb Hemost 2017;23(06):652-656

10 Berkouk-Redjimi Y, Ouarlent Y, Handi S, et al. Orthopaedic status and surgical needs of patients with haemophilia in Algeria. Open J Hematol 2013;4:1

11 The World Bank. Changes in country classifications. 2011. Available at: https://datahelpdesk.worldbank.org/knowledgebase/articles/378832 -what-is-the-world-bank-atlas-method. Accessed August 30, 2017

12 Gupta N, Belhani M, Benbouzid A, et al. The HAEMOcare protocol - a composite method to measure the disease burden from haemophilia in developing countries. Haematologica 2013;98 (Suppl 1):436-437

13 EuroQol Group. EuroQol-a new facility for the measurement of health-related quality of life. Health Policy 1990;16(03): 199-208

14 Blanchette VS, Key NS, Ljung LR, Manco-Johnson MJ, van den Berg HM, Srivastava A; Subcommittee on Factor VIII, Factor IX and Rare 
Coagulation Disorders of the Scientific and Standardization Committee of the International Society on Thrombosis and Hemostasis. Definitions in hemophilia: communication from the SSC of the ISTH. J Thromb Haemost 2014;12(11):1935-1939

15 Molho P, Rolland N, Lebrun T, et al. Epidemiological survey of the orthopaedic status of severe haemophilia A and B patients in France. The French Study Group. Haemophilia 2000;6(01): 23-32

16 Nilsson IM, Berntorp E, Löfqvist T, Pettersson H. Twenty-five years' experience of prophylactic treatment in severe haemophilia A and B. J Intern Med 1992;232(01):25-32

17 Bladen M, Main E, Hubert N, Koutoumanou E, Liesner R, Khair K. Factors affecting the Haemophilia Joint Health Score in children with severe haemophilia. Haemophilia 2013;19(04): 626-631

18 Manco-Johnson MJ, Abshire TC, Shapiro AD, et al. Prophylaxis versus episodic treatment to prevent joint disease in boys with severe hemophilia. N Engl J Med 2007;357(06):535-544

19 Taki M, Shirahata A. Current situation of regular replacement therapy (prophylaxis) for haemophilia in Japan. Haemophilia 2009;15(01):78-82

20 Biss TT, Chan AK, Blanchette VS, Iwenofu LN, McLimont M, Carcao MD; Association of Hemophilia Clinic Directors of Canada (AHCDC); Canadian Association of Nurses in Hemophilia Care (CANHC). The use of prophylaxis in 2663 children and adults with haemophilia: results of the 2006 Canadian national haemophilia prophylaxis survey. Haemophilia 2008;14(05):923-930

21 Fischer K, Astermark J, van der Bom JG, et al. Prophylactic treatment for severe haemophilia: comparison of an intermediate-dose to a high-dose regimen. Haemophilia 2002;8(06):753-760

22 Kalnins W, Mondorf W. Self-assessment of health and social status with emphasis on orthopedic function. Paper presented at: 37th Haemophilia Symposium; 2006; Hamburg, Germany

23 Berntorp E, Dolan G, Hay C, et al. European retrospective study of real-life haemophilia treatment. Haemophilia 2017;23(01):105-114

24 Saxena K. Barriers and perceived limitations to early treatment of hemophilia. J Blood Med 2013;4:49-56

25 duTreil S. Physical and psychosocial challenges in adult hemophilia patients with inhibitors. J Blood Med 2014;5:115-122

26 Srivastava A, Brewer AK, Mauser-Bunschoten EP, et al; Treatment Guidelines Working Group on Behalf of The World Federation Of Hemophilia. Guidelines for the management of hemophilia. Haemophilia 2013;19(01):e1-e47

27 World Federation of Hemophilia. World Federation of Hemophilia report on the Annual Global Survey 2013. Available at: http://www1. wfh.org/publications/files/pdf-1591.pdf. Accessed July 31, 2017

28 World Federation of Hemophilia. World Federation of Hemophilia report on the Annual Global Survey 2015. Available at: http:// www1.wfh.org/publications/files/pdf-1669.pdf. Accessed July 31, 2017 